\title{
Mapping the care process of moderate COVID-19 cases: the case of El-Mahalla chest hospital
}

\author{
Haytham S. Hassan \\ Assistant lecturer of accounting \\ Faculty of commerce - Tanta university \\ Haytham_saad@commerce.tanta.edu.eg
}




\title{
Mapping the care process of moderate COVID-19 cases: the case of El-Mahalla chest hospital
}

\begin{abstract}
Objective: this article aims at contributing to the attempts of costing COVID-19 patients in Egyptian quarantine hospitals (EQHs) by examining the process of delivering healthcare for moderate COVID-19 cases in El-Mahalla chest hospital (MCH) as one among several hospitals designated by the Egyptian ministry of health and population (MoHP) to serve the needs of COVID-19 patients.

Methods: as a moderate COVID-19 case myself, I was quarantined in MCH for six days where I relied on observations and interviews to identify the care process of moderate COVID-19 cases including activities, tasks, actions, resources consumed, and supplies used.

Conclusion: defining the healthcare process of COVID-19 cases would enable TD-ABC to estimate care costs, thereby narrowing the gap between cost and outcomes studies in the Egyptian environment which would allow cost teams to work alongside medical teams to identify sustainable improvements and deliver valuable care.
\end{abstract}

Keywords: COVID-19, TD-ABC, Patient Flow, Cost, Process Mapping.

\section{Background:}

On February 14th, 2020, Egyptian ministry of health and population (MoHP), announced officially the arrival of COVID-19 pandemic in the country, by confirming its first case ${ }^{1}$ of the deadly virus. As a result, Egyptian healthcare system has started establishing a list of hospitals to quarantine infected cases. Consequently, clinical teams began to interact directly with COVID-19 patients on a daily basis allowing them to conduct numerous studies on the medical aspects of COVID-19 such as, symptoms, complications, health outcomes, vaccine development ... etc. However, valuable care is not just about health outcomes, it is a two-dimensional concept, which also includes costs along with health outcomes. In this context, value of care can be defined as "health outcomes achieved relative to costs of care incurred" (Porter, 2010), and while clinical studies have focused on estimating the outcomes part, limited interaction with the care process of COVID-19 patients has prevented cost studies from keeping pace with their clinical counterparts which resulted in delays regarding estimating the cost part and thus a gap between cost and outcomes studies.

On May 29th, 2020, I began to develop COVID-19 symptoms, however, I never isolated myself, consequently, the virus spread to my family and it was not long before we were having chest pains, so we decided to seek help. As a result, we were assigned two quarantine rooms in El-Mahalla chest hospital $(\mathrm{MCH})$ where I observed the care process

\footnotetext{
${ }^{1}$ The terms "case" and "patient" will be used interchangeably throughout the text.
} 
of COVID-19 patients. accordingly, this article aims to narrow the cost-outcomes gap by mapping the flow of moderate COVID-19 cases in $\mathrm{MCH}$ which would bring us one step closer toward estimating healthcare costs of COVID-19 cases.

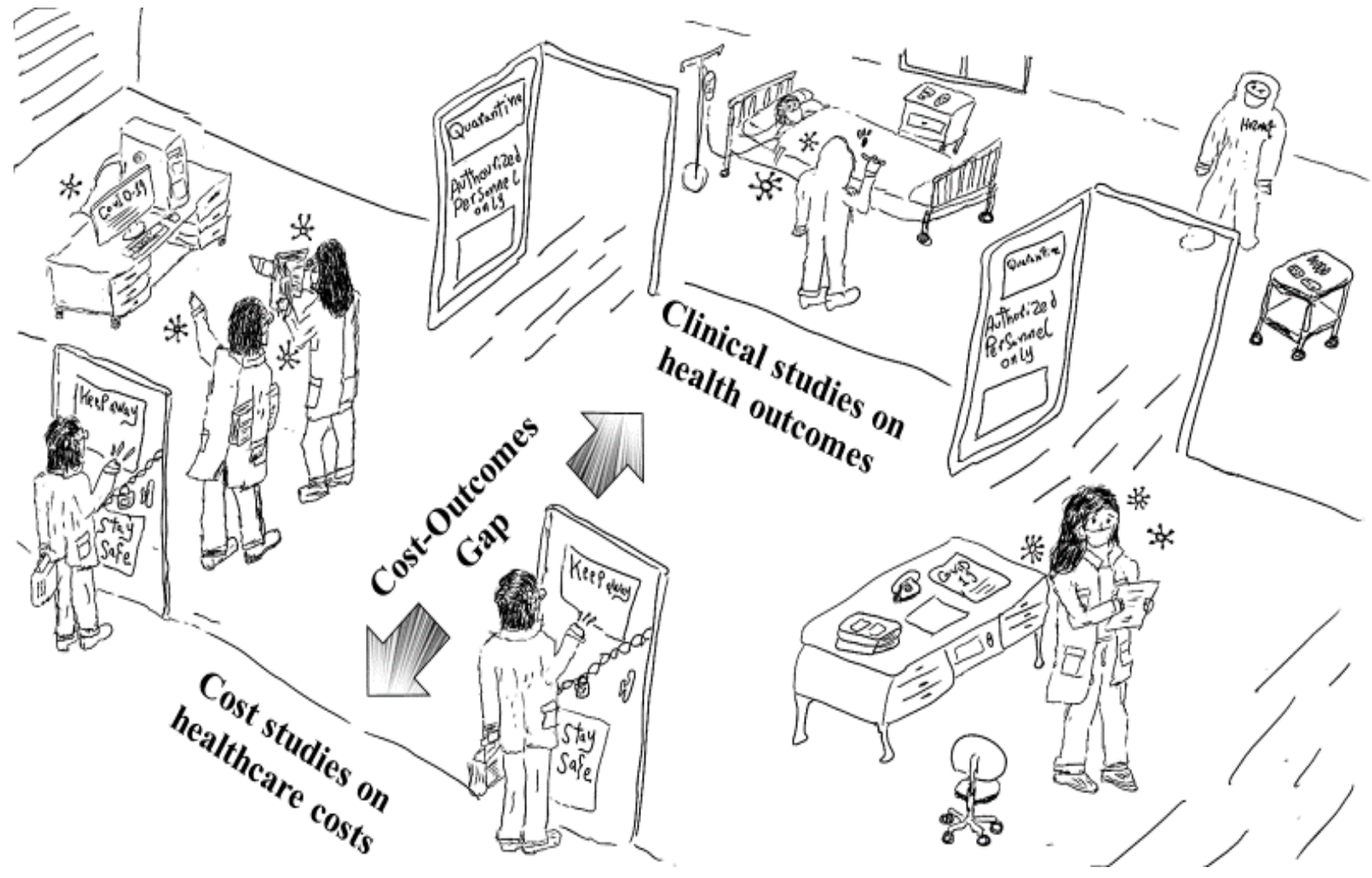

Figure 1 Cost-outcomes gap

\section{About El-Mahalla Chest Hospital (MCH):}

El-Mahalla chest hospital (MCH) was established in 1948 to provide healthcare for tuberculosis cases and is considered by many to be the second largest chest hospital in Egypt preceded only by Abassya chest diseases hospital. Upon the spread of COVID-19 in Egypt, MoHP has listed MCH as one among several Egyptian quarantine hospitals (EQHs) dedicated to serving COVID-19 cases. MCH contains five separate main buildings, which are: inpatient clinics, reception and outpatient clinics, laboratories, Asthma and allergy, and finally, the inventory (Dora \& Adel, 2020).

\section{Classification of COVID-19 patients:}

In this article, I will rely on the work of (Gülşen, 2020) who developed a simple classification of COVID-19 patients. In this classification, COVID-19 patients ranged from asymptomatic cases to very severe/critical cases, while moderate cases were described as: patients requiring hospitalization, with lobar or multi-lobar pneumonia with/without the need for supplemental oxygen, or refractory to initial treatment. The previous description of moderate COVID-19 cases matches the majority of infected cases that were found in the quarantine wards of $\mathrm{MCH}$ at the time of my admission. 


\section{Flow of moderate COVID-19 cases in MCH:}

For simplicity, I will divide the care process of moderate COVID-19 cases into three different phases each representing one or more activities, these phases are: arrival, care, and discharge. On one hand, phase I (arrival) will focus on diagnostic activities performed before admission, while on the other hand, phase II (care) will discuss how the care process proceeds and goes on, and lastly, phase III (discharge) will concentrate on activities performed before leaving $\mathrm{MCH}$. It goes without saying that every activity contains a series of tasks which in turn require a set of actions to be performed. Each phase is discussed below in detail:

\section{Phase I-Arrival:}

Upon arrival, patients are informed that they should get examined first before admission, in order to confirm their COVID-19 infection status and identify the severity of infection. To do so, a single diagnostic procedure is carried out, which is a chest computed tomography (CT) scan. The chest CT scan takes a standard time of up to 10 minutes and is performed in the CT unit by a radiologist, a radiology technician, and of course a CT scanner.

Once infection is confirmed and its severity identified, infected patients are permitted to register for admission. During registration, patients are usually required to provide the receptionist with a photocopy of the ID card, the names and phone numbers of two relatives or friends so that the hospital can communicate with them in case of emergencies, in addition to some basic information such as name, age, profession, complications, the existence of any other diseases ... etc., Each patient spends around 12 minutes finishing all the needed paperwork.

Afterwards, patients are classified according to their COVID-19 infection severity into mild, moderate, and severe \& critical cases. Mild cases are discharged directly for home isolation, while moderate cases are admitted to the quarantine wards of the hospital, and ultimately, severe \& critical COVID-19 cases are admitted directly to the intensive care unit (ICU).

\section{Phase II-Care:}

Providing healthcare for moderate COVID-19 cases starts by assigning a quarantine room for each patient to stay in till discharge. a few minutes later, a lab technician pays each patient a visit for approximately 5 minutes to collect the required specimens for polymerase chain reaction (PCR) test. Afterwards, collected specimens are sent to the $\mathrm{lab}^{2}$ for testing.

\footnotetext{
${ }^{2}$ Further examination is required to determine how activities are carried out in other operating units, such as the laboratory, the housekeeping department, and the kitchen. I propose using time-driven activity-based costing (TD$\mathrm{ABC}$ ) to develop a capacity cost rate and a time equation (because it won't be cost effective to use process maps) for each operating unit.
} 
Hereafter, most tasks are carried out frequently and are categorized into housekeeping ${ }^{3}$ tasks and medical tasks. Given below is a chronological list of these tasks which are performed in each quarantine room:

1- A meal is provided for each case, three times a day. The meal varies depending on whether it is for breakfast, lunch, or dinner.

2- For safety purposes, a housekeeper is assigned to clean and sanitize each room for about 1 minute (including food delivery), twice daily.

3- Every day, three routine-checkup visits are paid by nurses to the quarantined cases, each for about 5 minutes. Actions performed by nurses during each visit are almost identical, however, some of the medications provided for each case vary between cases based on several factors including but not limited to age, complications, severity of illness, development of new symptoms ... etc., and aside from those medications, almost everything is identical. For simplicity, I will divide the abovementioned actions into two categories, recurring (performed each visit) and non-recurring (performed once or twice daily or in certain days), recurring actions include:

a) Measuring oxygen saturation using a pulse oximeter and temperature using a disposable thermometer,

b) Providing two tablets, which are: clarithromycin (500mg), and multivitamin,

While non-recurring actions include:

a) Injecting cefoperazone (1gm), twice daily,

b) Injecting anticoagulant, once per day,

c) Providing a tablet of paracetamol $(500 \mathrm{mg})$, twice daily, or based on patient's temperature,

d) Providing a tablet of hydroxychloroquine $(400 \mathrm{mg})$, once daily, for certain number of days,

e) Inserting an intravenous (IV) cannula to facilitate injecting cefoperazone and anticoagulant,

f) And lastly, providing a cough syrup bottle.

4- Every two days, A visit is paid by the pulmonologist to each quarantined case for about 2 minutes to determine the health status of each case, whether the case suffers any side effects due to medications, and to identify the progress each patient makes toward recovery.

\section{Possible additional activities and tasks during Phase II-Care:}

The length of this phase may vary between patients, as Additional activities and tasks might be required for some cases depending on several factors, such as, initial health status, age, complications, comorbidities, and severity of COVID-19. For instance, some cases have

\footnotetext{
${ }^{3}$ Housekeepers are entrusted with both cleaning and food delivery tasks.
} 
been provided with supplemental oxygen in their quarantine rooms, while some others have been transferred to the ICU to get them connected to ventilators.

At the end of phase II, moderate COVID-19 cases often take different paths depending on case stability or severity. Stable cases (recovered and mild cases) are discharged for home isolation, while severe \& critical cases are transferred to the ICU.

\section{Phase III-Discharge:}

Recovered COVID-19 patients are informed by nurses that their health status has been improved, and that they must be discharged from the hospital to allow admission of new cases. Before leaving the hospital, recovered patients are given a list of prescribed medications relevant to their condition and are advised to isolate themselves at home for at least 14 days. Figure 2 below shows the care process of COVID-19 cases in MCH:

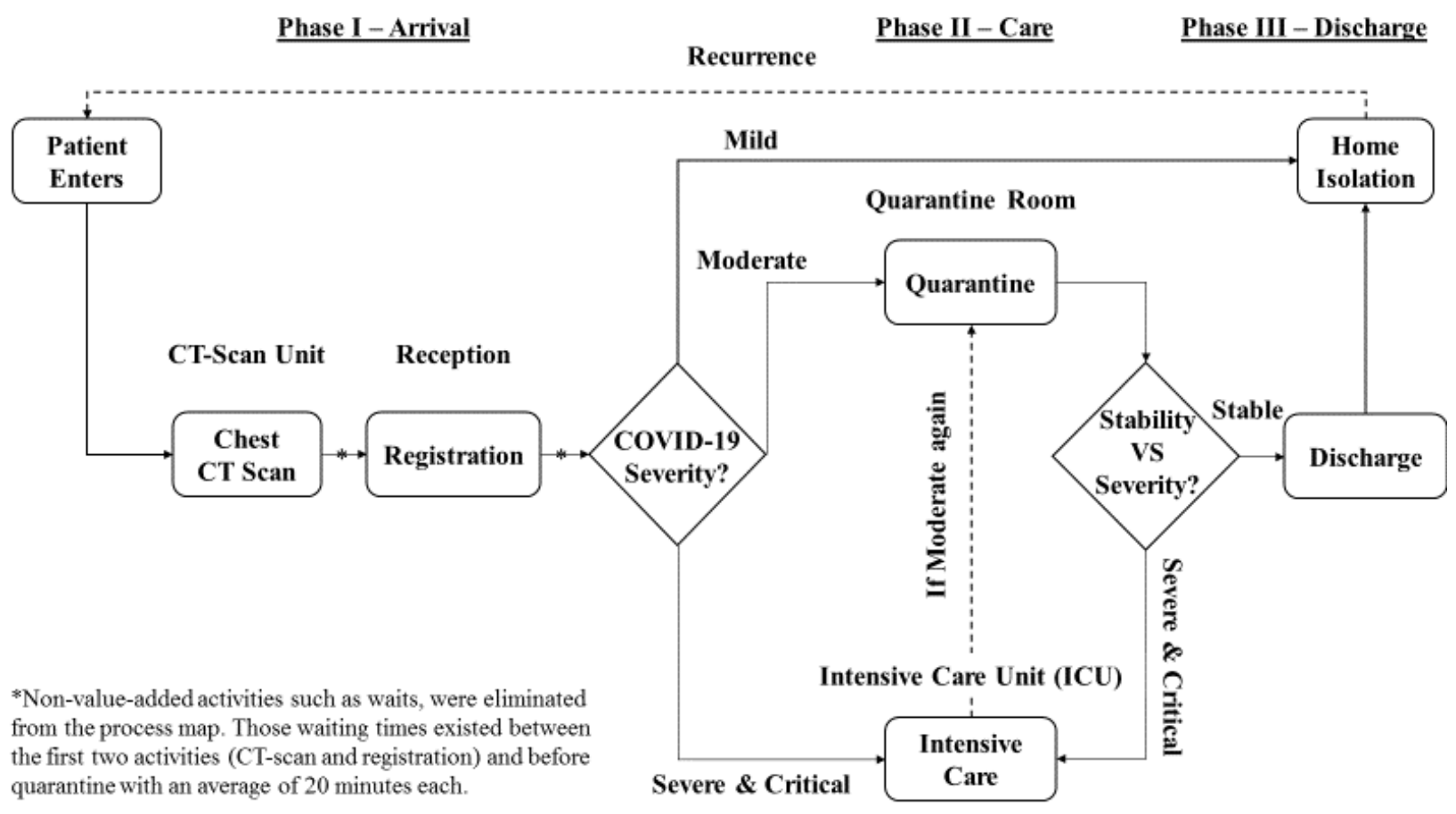

Figure 2 The care process of COVID-19 patients in MCH

\section{Costing moderate COVID-19 cases:}

Since the arrival of COVID-19 in Egypt, many Egyptian private hospitals have shifted their focus to providing healthcare services for COVID-19 patients as a means to increase revenues and have relied on the specialty per-diem basis to determine the prices of healthcare services. The specialty per-diem basis sets equal daily cost rates for specific hospital departments (mainly inpatient clinics for mild and moderate COVID-19 cases, and ICU for severe and critical COVID-19 cases) regardless of resource consumption variations between patients which would lead to overcharging or undercharging patients (Azoulay, et al., 2007). Usage of such traditional costing approaches (i.e. specialty per- 
diem) reflects low awareness of advanced costing practices and of the usefulness of information derived from them.

Recently, Time-Driven Activity-Based Costing (TD-ABC) has been widely used to measure costs of care in multiple hospitals. In doing so, TD-ABC utilizes process mapping to identify the mix of resources used in delivering care. Accordingly, identifying the care process of COVID-19 patients would allow TD-ABC to estimate costs of care, thereby providing more relevant cost information to help improve healthcare outcomes and manage healthcare costs.

DAILY COST PER QUARANTINE ROOM
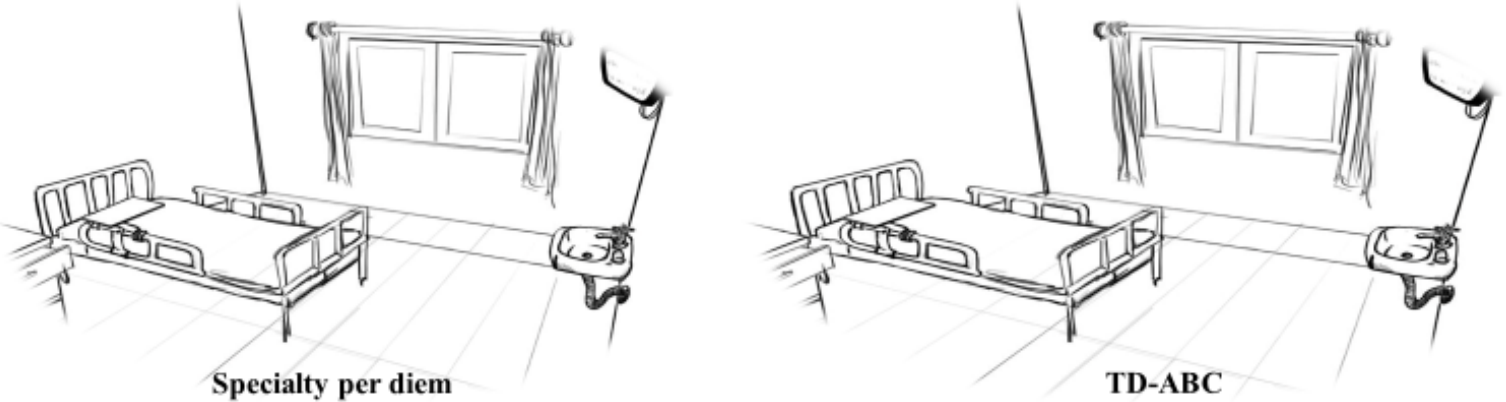

Specialty per diem rate $=\frac{\text { Total departmental costs }}{\text { Number of days } * * *}$

Identificationsand notes:

$\mathrm{X}_{\mathrm{b}}$ : Time Spent in Space $\mathrm{b}$, where $\mathrm{b}=1,2, \ldots \mathrm{B}$

$\mathrm{P}_{\mathrm{k}}$ : CCR of Personnel $\mathrm{k}$, where $\mathrm{k}=1,2, \ldots \mathrm{K}$

$E_{j}$ : CCR of Equipment $i$, where $i=1,2, \ldots I$

$\mathrm{S}_{\mathrm{b}}$ : CCR of Space $b$, where $\mathrm{b}=1,2, \ldots \mathrm{B}$

$C_{j}$ : Cost of Supplies Consumed in Activity $j$, where $j=1,2, \ldots \mathrm{n}$

$T_{j}$ : Cost of Activity $j$, where $j=1,2, \ldots n$

*CCR: Capacity Cost Rate

** These costs are considered only in the first hospitalization day and excluded thereafter.

***Cost driver is the length of stay (LoS)

\begin{tabular}{|c|c|c|c|}
\hline Resources & CCR $^{*}$ & Time & Cost \\
\hline Quarantine Room & $S_{D 3}$ (Daily CCR) & $1_{\text {Day }}$ & \\
\hline Lab Technician** & $P_{4}$ & $5_{\text {Minutes }}$ & \\
\hline Laboratory (Testing)** & $S_{4}$ & $X_{4}$ & \\
\hline Kitchen (Meal Preparation) & $S_{5}$ & $X_{5}$ & \\
\hline Nurse & $P_{5}$ & $\mathbf{1 5}_{\text {Minutes }}$ & \\
\hline Housekeeper & $P_{6}$ & $1_{\text {Minutes }}$ & \\
\hline Pulmonologist & $P_{7}$ & $1_{\text {Minutes }}$ & \\
\hline Consumables (Pills \& Medications, Cannulas, Thermometers, Syringes, ... etc) & $C_{3}$ \\
\hline \multicolumn{3}{|c|}{ Total Activity Cost } & $T_{3}$ \\
\hline
\end{tabular}

$T_{3}=1 S_{D 3}+5 P_{4}+S_{4} X_{4}+S_{5} X_{5}$ $+15 P_{5}+1 P_{6}+1 P_{7}+C_{3}$

Figure 3 Comparison between specialty per diem basis and TD-ABC approach ${ }^{4}$

Unlike the specialty per-diem basis, TD-ABC considers disease severity and hence resource consumption in estimating costs, thereby allowing hospitals to make relevant pricing decisions based on resource consumption.

\section{Integrating TD-ABC with COVID-19 patient flow:}

Mapping the care process of COVID-19 cases in MCH can support TD-ABC by identifying the medical and administrative activities performed on COVID-19 patients (Kaplan \& Witkowski, 2014), which would facilitate:

1- Tracking resource consumption,

2- Providing a basis, over which pricing decisions of healthcare services for COVID19 cases can be made,

\footnotetext{
${ }^{4}$ Special thanks to the designer Gab Nascimento who granted me the permission to use the art depicted in Figure 4, retrieved from: https://www.deviantart.com/tsu-gambler/art/Hospital-room-316549039
} 
3- And finally, improving care value by identifying potential opportunities to reduce care costs without impacting healthcare outcomes negatively (Kaplan \& Witkowski, 2014).

Figure 4 shows how TD-ABC can utilize process mapping in determining care costs of moderate COVID-19 cases:

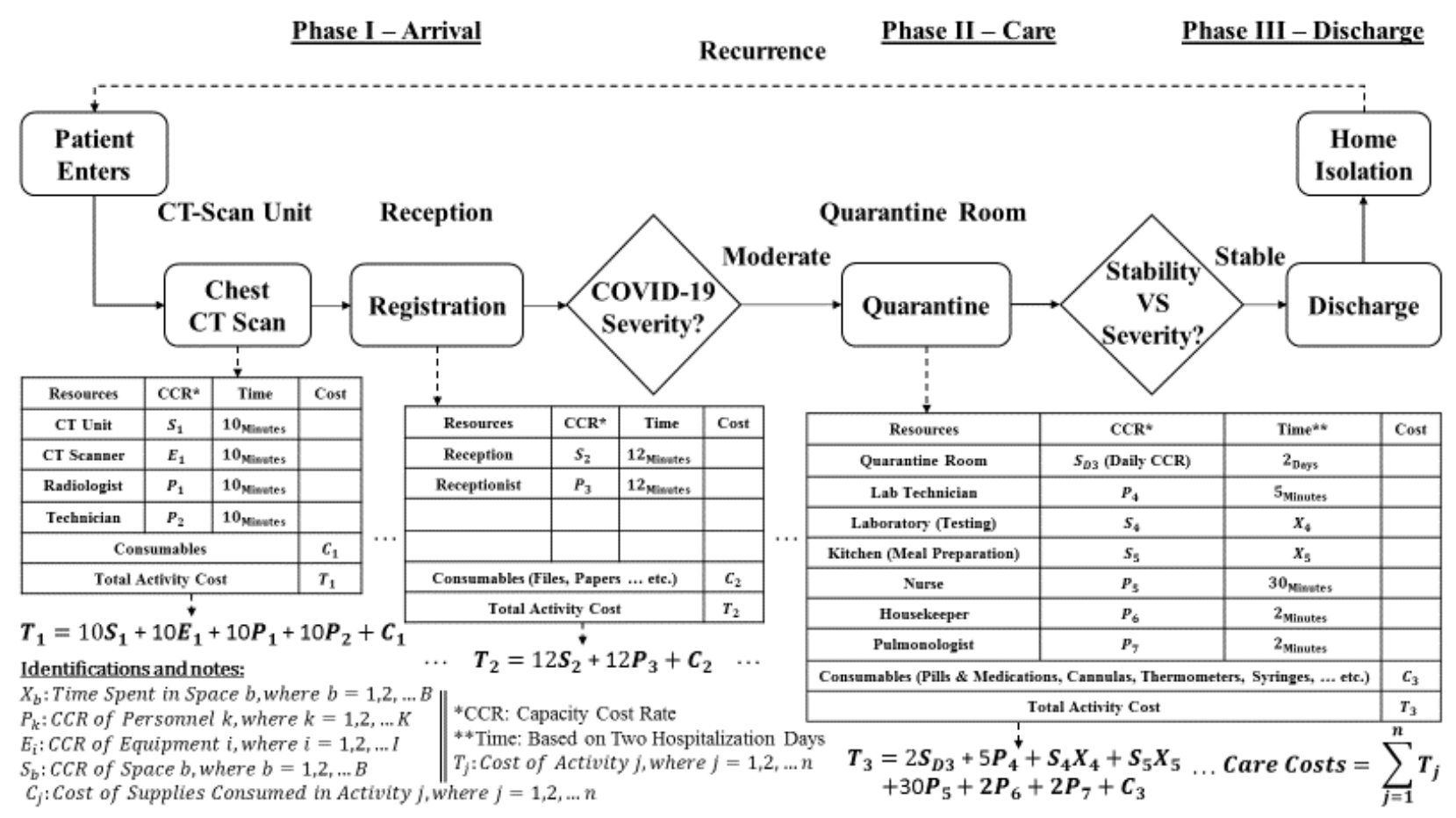

Figure 4 integrating process maps into TD-ABC

\section{Future research opportunities in $\mathrm{MCH}$ :}

This article aimed at shedding some light on the care process of COVID-19 patients in EQHs by identifying different activities, tasks and actions performed throughout the care process. The information provided in this article can benefit both management accountants and clinicians in:

1- Evaluating the hospital degree of compliance with the applicable Egyptian national guidelines for COVID-19 at that time (Wadie, Elsayed, Elnagar, Abdelzaher, \& Essa, 2020),

2- Developing CCRs and time equations for unexamined operating units such as the lab, the housekeeping department, and the kitchen.

3- Estimating care costs of moderate COVID-19 cases using TD-ABC, by obtaining cost information from the records of $\mathrm{MCH}$,

4- Making further examinations of activities, tasks and actions performed on severe and critical COVID-19 cases in ICU, 
5- Determining how the information derived from TD-ABC could be used as inputs to several other management accounting techniques, leading to increased value of care in $\mathrm{MCH}$,

6- Providing the hospital with improvement recommendations, by examining the overall care delivery value chain of COVID-19 patients. For example, patients had to interact with the receptionist several times during registration. However, reducing the interaction of COVID-19 patients with employees may reduce infection rates among hospital administrative staff, which may require additional examination of the hospital admission system.

\section{Conclusion:}

Identifying the process of delivering healthcare to COVID-19 patients including activities, tasks, actions, resources used, and supplies consumed would help estimating healthcare costs, thus narrowing the cost-outcomes gap and allowing cost teams to work alongside medical teams to deliver valuable care.

\section{Acknowledgment:}

I gratefully thank all the hard-working staff in El-Mahalla chest hospital for all their contributions and my family for all their contributions and support.

\section{References:}

Azoulay, A., Doris, N. M., Filion, K. B., Caron, J., Pilote, L., \& Eisenberg, M. J. (2007). The use of the transition cost accounting system in health services research. Cost Effectiveness and Resource Allocation, 5(1), 1-9.

Dora, A., \& Adel, M. (2020, January 15). Watch the second oldest chest hospital established by king Farouk: its modernizing cost is 40 million. Retrieved March 2, 2021, from Youm7: http://www.youm7.com/4584357

Gülşen, A. (2020). Simple classification of COVID-19 patients. J Lung Pulm Respir Res, $7(3), 62-63$.

Kaplan, R. S., \& Witkowski, M. L. (2014). Better accounting transforms health care delivery. Accounting Horizons, 28(2), 365-383.

Porter, M. E. (2010). What is value in health care. N Engl J Med, 363(26), 2477-2481.

Wadie, S., Elsayed, M. M., Elnagar, I. H., Abdelzaher, S. R., \& Essa, O. H. (2020). Egyptian National Guidelines for COVID-19. Retrieved March 4, 2021, from hiph.alexu.edu.eg: https://hiph.alexu.edu.eg/images/egyptian_national_guidelines_covid19.pdf.pdf.pdf 